\title{
A Calculation of the Culler-Shalen Seminorms Associated to Small Seifert Dehn Fillings
}

\author{
Leila Ben Abdelghani and Steven Boyer ${ }^{1}$
}

\section{Introduction}

Throughout this paper $M$ will be a compact, connected, irreducible, orientable 3manifold whose boundary is a torus. We shall fix a base point in $\partial M$ and therefore have a well-defined homomorphism $\pi_{1}(\partial M) \rightarrow \pi_{1}(M)$.

The $S L(2, \mathbb{C})$-representation variety of $\pi_{1}(M)$ is a complex affine algebraic variety $R(M)$ whose points correspond to representations of $\pi_{1}(M)$ with values in $S L(2, \mathbb{C})$ [9]. If $\operatorname{tr}$ : $S L_{2}(\mathbb{C}) \rightarrow \mathbb{C}$ denotes the trace function, then each $\rho \in R(M)$ determines

$$
\chi_{\rho}: \pi_{1}(M) \rightarrow \mathbb{C} \quad \chi_{\rho}(\gamma)=\operatorname{tr}(\rho(\gamma))
$$

called its character, and the set of such characters $X(M)=\left\{\chi_{\rho} \mid \rho \in R(M)\right\}$ admits the structure of a complex affine algebraic variety in such a way that the function

$$
t: R(M) \rightarrow X(M), \quad \rho \mapsto \chi_{\rho}
$$

is regular [9]. It turns out that $t$ can be canonically identified with the algebro-geometric quotient of $R(M)$ by the natural action of $S L(2, \mathbb{C})$ [13, Theorem 3.3.5]. This means that $\mathbb{C}[X(M)]$ is isomorphic to the ring of invariants $\mathbb{C}[R(M)]^{S L(2, \mathbb{C})}$ and $t$ corresponds to the inclusion $\mathbb{C}[R(M)]^{S L(2, \mathbb{C})} \subset \mathbb{C}[R(M)]$. The orbit of a representation $\rho$ under this action will be denoted by $\mathcal{O}(\rho)$.

Each $\gamma \in \pi_{1}(M)$ determines an element of the coordinate ring $I_{\gamma} \in \mathbb{C}[X(M)]$ given by evaluation: $I_{\gamma}(\chi)=\chi(\gamma)$. Of more interest to us is the related function

$$
f_{\gamma}(\chi)=\chi(\gamma)^{2}-4
$$

Research has shown that many topological properties of a 3-manifold $M$, as above, are encoded in the functions $f_{\gamma}$, so it is of interest to study their basic properties. One of the goals of this paper is to find a useful set of conditions which allow us to determine the multiplicities of certain characters as zeros of the functions $f_{\gamma}$ when $\gamma \in \pi_{1}(\partial M)$. In order to make sense of this statement, we introduce several notions.

\footnotetext{
${ }^{1}$ Partially supported by grants: NSERC OGP 0009446 and FCAR EQ 3518.

Key words. $S L(2, \mathbb{C})$-character variety, Culler-Shalen seminorm, Dehn filling, small Seifert manifold. 1980 Mathematics Subject Classification (1991 Revision). Primary 57M05, 57R65
} 
A class in $\alpha \in H_{1}(\partial M)$ determines an element $\gamma(\alpha) \in \pi_{1}(M)$ through the Hurewicz isomorphism $H_{1}(\partial M) \cong \pi_{1}(\partial M) \rightarrow \pi_{1}(M)$. We shall abuse notation by writing $\alpha$ for $\gamma(\alpha)$. For instance the function $f_{\gamma(\alpha)}$ will be denoted by $f_{\alpha}$.

A slope on $\partial M$ is a $\partial M$-isotopy class of essential, simple closed curves on $\partial M$. Any slope $r$ on $\partial M$ determines (and is determined by) a pair $\pm \alpha(r)$ of primitive elements of $H_{1}(\partial M)$ - the images in $H_{1}(\partial M)$ of the two generators of $H_{1}(C) \cong \mathbb{Z}$ where $C \subset \partial M$ is a representative curve for $r$.

Consider a non-trivial curve $X_{0} \subset X(M)$, that is a curve which contains the character of an irreducible representation. Assume that $X_{0}$ is irreducible. There is a unique 4dimensional subvariety $R_{0} \subset R(M)$ for which $t\left(R_{0}\right)=X_{0}$ (cf. [5, Lemma 4.1]). The smooth projective model $\tilde{X}_{0}$ of $X_{0}$ decomposes as

$$
X_{0} \stackrel{\nu}{\longleftarrow} X_{0}^{\nu} \stackrel{i}{\longrightarrow} X_{0}^{\nu} \cup \mathcal{I}=\tilde{X}_{0}
$$

where $\nu: X_{0}^{\nu} \rightarrow X_{0}$ is a surjective regular birational equivalence, $i$ is an inclusion, and $\mathcal{I}$ is the finite set of ideal points of $X_{0}$. These maps induce an isomorphism between function fields:

$$
\mathbb{C}\left(X_{0}\right) \rightarrow \mathbb{C}\left(\tilde{X}_{0}\right), \quad f \mapsto \tilde{f} .
$$

We use $Z_{x}\left(\tilde{f}_{\gamma}\right)$ to denote the multiplicity of $x \in \tilde{X}_{0}$ as a zero of $\tilde{f}_{\gamma}$. By convention this means that $Z_{x}\left(\tilde{f}_{\gamma}\right)=\infty$ if $\tilde{f}_{\gamma}=0$. The motivation to investigate these quantities arises when $\gamma=\alpha(r)$ for some slope $r$ on $\partial M$. Indeed, if $M(r)$ is the 3-manifold obtained by Dehn filling $M$ along $r$, there is a quantifiable relationship between $Z_{x}\left(\tilde{f}_{\alpha(r)}\right)$ and the $\operatorname{PSL}(2, \mathbb{C})$ representation theory of $\pi_{1}(M(r))$ [8], [4,5,7]. For instance from [8, Proposition 1.5.4] it follows that if $x \in X_{0}^{\nu}, \beta \in \pi_{1}(\partial M)$, and

$$
Z_{x}\left(\tilde{f}_{\beta}\right)<Z_{x}\left(\tilde{f}_{\alpha(r)}\right)<\infty
$$

then $\rho(\alpha(r))= \pm I$ for each $\rho \in R_{0}$ whose character is $\nu(x)$. Hence each such $\rho$ induces a representation $\pi_{1}(M(r)) \rightarrow P S L(2, \mathbb{C})$. Under appropriate hypotheses, more can be said. For instance if $\pi_{1}(M(r))$ is a finite group, then $Z_{x}\left(\tilde{f}_{\alpha(r)}\right)=Z_{x}\left(\tilde{f}_{\beta}\right)+2$ [4]. These facts were essential ingredients in the proofs of the cyclic surgery theorem [8] and the finite surgery theorem [7]. Theorem A below allows us to quantify the differences $Z_{x}\left(\tilde{f}_{\alpha(r)}\right)-Z_{x}\left(\tilde{f}_{\beta}\right)$ in more general and topologically significant circumstances.

A representation $\rho \in R(M)$ determines a homomorphism $A d \circ \rho: \pi_{1}(M) \rightarrow \operatorname{Aut}\left(s l_{2}(\mathbb{C})\right)$ where $A d: S L(2, \mathbb{C}) \rightarrow A u t\left(s l_{2}(\mathbb{C})\right)$ is the adjoint representation. We shall simplify notation by writing $H^{1}(M ; A d \rho)$ for the cohomology group $H^{1}\left(M ; s l_{2}(\mathbb{C})_{A d \circ \rho}\right)$. Note that if $\rho(\alpha(r))= \pm I$ for some slope $r$, then $A d \circ \rho$ induces a representation $\pi_{1}(M(r)) \rightarrow P S L(2, \mathbb{C})$. In particular it makes sense to consider the cohomology group $H^{1}(M(r) ; A d \rho)$. 
Theorem A Fix a slope $r$ on $\partial M$ and consider a non-trivial, irreducible curve $X_{0} \subset$ $X(M)$. Suppose that $x \in X_{0}^{\nu}$ is such that $\nu(x)=\chi_{\rho}$ for some representation $\rho \in R_{0}$ with non-abelian image which satisfies $\rho(\alpha(r)) \in\{ \pm I\}$. Assume that $H^{1}(M(r) ; A d \rho)=0$ and $\rho\left(\pi_{1}(\partial M)\right) \not \subset\{ \pm I\}$.

(1) If $\beta \in \pi_{1}(\partial M)$ and $\rho(\beta) \neq \pm I$, then

$$
Z_{x}\left(\tilde{f}_{\alpha(r)}\right) \geq Z_{x}\left(\tilde{f}_{\beta}\right)+2 .
$$

(2) If $\beta \in \pi_{1}(\partial M)$ and $Z_{x}\left(\tilde{f}_{\alpha(r)}\right)>Z_{x}\left(\tilde{f}_{\beta}\right)$, then $f_{\alpha(r)} \mid X_{0} \neq 0, \rho(\beta) \neq \pm I$, and

$$
Z_{x}\left(\tilde{f}_{\alpha(r)}\right)=Z_{x}\left(\tilde{f}_{\beta}\right)+2 .
$$

We can be a little more specific when $\rho\left(\pi_{1}(\partial M)\right)$ is diagonalisable, for then it is clear that $Z_{x}\left(\tilde{f}_{\beta}\right)=0$ whenever $\rho(\beta) \neq \pm I$. Hence under the hypotheses of Theorem A (2) we have $Z_{x}\left(\tilde{f}_{\alpha(r)}\right)=2$. In the case where $\rho\left(\pi_{1}(\partial M)\right)$ is parabolic subgroup, $Z_{x}\left(\tilde{f}_{\beta}\right)>0$ for each $\beta \in H_{1}(\partial M)$. When $f_{\alpha(r)} \mid X_{0} \neq 0$ we know of no instance where $Z_{x}\left(\tilde{f}_{\alpha(r)}\right)>3$ (cf. [3], $[12])$.

Theorem A begs the question: given a non-trivial curve $X_{0} \subset X(M)$ and some $x \in X_{0}^{\nu}$, when is there a representation $\rho \in R_{0}$, with non-abelian image, such that $\chi_{\rho}=\nu(x)$ ? According to $[2, \S 2]$, such a representation exists if and only if $\nu(x)$ is a non-trivial character, that is, a character whose image is not contained in $\{ \pm 2\}$. If $\operatorname{rank}_{\mathbb{Z}} H_{1}(M)=1$, then each character in $X_{0}$ is non-trivial ([2, Proposition 2.8]).

Corollary B Assume the hypotheses of Theorem A.

(1) If $\beta \in H_{1}(\partial M) \backslash\{0\}$ and $f_{\beta} \mid X_{0}=0$, then $f_{\alpha(r)} \mid X_{0}=0$.

(2) If $f_{\alpha(r)} \mid X_{0} \neq 0$ and $\beta \in H_{1}(\partial M)$, then

$$
Z_{x}\left(\tilde{f}_{\beta}\right)= \begin{cases}Z_{x}\left(\tilde{f}_{\alpha(r)}\right)-2 & \text { if } \beta \in H_{1}(\partial M) \backslash\{n \alpha(r) \mid n \in \mathbb{Z}\} \\ Z_{x}\left(\tilde{f}_{\alpha(r)}\right) & \text { if } \beta=n \alpha(r) \text { some } n \in \mathbb{Z} \backslash\{0\} \\ \infty & \text { if } \beta=0 .\end{cases}
$$

In order to describe a criterion on the manifold $M(r)$ which allows us to apply the results above in situations of interest, consider the small Seifert manifolds, i.e. those 3manifolds which admit the structure of a Seifert fibred space whose base orbifold is the 2 -sphere with at most three cone points. If we exclude $S^{1} \times S^{2}$, these manifolds are known to be irreducible. Further they are Haken if and only if they have infinite first homology [11, VI.13]. For us, their key property is contained in the following proposition.

Proposition Suppose $W$ is a small Seifert manifold and $\rho \in \operatorname{Hom}\left(\pi_{1}(W), P S L(2, \mathbb{C})\right)$ is either irreducible or non-abelian. Then the cohomology group $H^{1}(W ; A d \rho)$ is zero. 
The proof of this proposition in the case where $\rho$ factors through $S L(2, \mathbb{C})$ can be found in [6]. The general result follows from a similar computation.

Consider a curve $X_{0} \subset X(M)$. The Culler-Shalen seminorm

$$
\|\cdot\|_{X_{0}}: H_{1}(\partial M ; \mathbb{R}) \rightarrow[0, \infty)
$$

was introduced in [8] to study Dehn fillings of $M$ with cyclic fundamental groups. For $\beta \in H_{1}(\partial M),\|\beta\|_{X_{0}}$ is the degree of $f_{\beta} \mid: X_{0} \rightarrow \mathbb{C}$, or equivalently

$$
\|\beta\|_{X_{0}}= \begin{cases}0 & \text { if } f_{\beta} \mid X_{0}=0 \\ \sum_{x \in \tilde{X}_{0}} Z_{x}\left(\tilde{f}_{\beta}\right) & \text { if } f_{\beta} \mid X_{0} \neq 0 .\end{cases}
$$

Roughly speaking, $\|\alpha(r)\|_{X_{0}}$ measures the number of characters in $X_{0}$ of representations which send $\alpha(r)$ to $\pm I$. As a consequence of Theorem A we are often able to give an explicit calculation of $\|\alpha(r)\|_{X_{0}}$ when $M(r)$ is small Seifert.

Theorem $\mathbf{C}$ Let $M$ be the exterior of a knot in a closed, connected, orientable 3-manifold $W$ for which $\operatorname{Hom}\left(\pi_{1}(W), P S L(2, \mathbb{C})\right)$ contains only diagonalisable representations. Suppose further that there is a non-boundary slope $r$ for which $M(r)$ is an irreducible, non-Haken small Seifert manifold. Fix a non-trivial, irreducible curve $X_{0} \subset X(M)$ for which $f_{\alpha(r)} \mid X_{0}$ is non-constant. Then

$$
\|\alpha(r)\|_{X_{0}}=m_{0}+2 A
$$

where $m_{0}=\sum_{x \in \tilde{X}_{0}} \min \left\{Z_{x}\left(\tilde{f}_{\alpha}\right)\left|\tilde{f}_{\alpha}\right| \tilde{X}_{0} \not \equiv 0\right\}$ and $A$ is the number of characters $\chi_{\rho} \in X_{0}$ of non-abelian representations $\rho \in R_{0}$ such that $\rho(\alpha(r))= \pm I$.

The condition that $\operatorname{Hom}\left(\pi_{1}(W), P S L(2, \mathbb{C})\right)$ contains only diagonalisable representations is a regularity condition. It implies that no representation in $\operatorname{Hom}\left(\pi_{1}(M), P S L(2, \mathbb{C})\right)$ which is non-diagonalisable can send $\pi_{1}(\partial M)$ into $\{ \pm I\}$. This ensures the smoothness of $X_{0}$ at certain characters (cf. Theorem 1.1). Typically $W$ will be $S^{3}$ or a lens space.

Though we have assumed above that $X_{0}$ is irreducible, this is not necessary. CullerShalen seminorms are defined for arbitrary curves in $X(M)$, i.e. curves which are possibly reducible (cf. $\S 6$ of [7]). A comparable proof shows that Theorem $\mathrm{C}$ holds in this more general situation. The interest in results of this form stems from the study of exceptional Dehn fillings of $M$. Such fillings are, on the whole, well understood, except for those that yield small Seifert manifolds. Calculations of the sort found in Theorem $\mathrm{C}$ were instrumental in the proofs of the cyclic surgery theorem [8] and the finite surgery theorem [7], as well as other results on exceptional fillings [5]. Our hope is that the result above will help shed some light on the general problem of small Seifert fillings. In that context, it is of interest to determine the number of $P S L(2, \mathbb{C})$-characters $(\S 2)$ of the fundamental group a small Seifert manifold. This we do in our last result. 
Suppose that $W$ is a non-Haken Seifert manifold whose base orbifold is of the form $S^{2}(p, q, r)$ where $p, q, r \geq 2$. There is a presentation of $\pi_{1}(W)$ of the form

$$
\pi_{1}(W)=<x, y, h \mid h \text { central, } x^{p}=h^{a}, y^{q}=h^{b},(x y)^{r}=h^{c}>
$$

where $\operatorname{gcd}(a, p)=\operatorname{gcd}(b, q)=\operatorname{gcd}(c, r)=1[11, \S \mathrm{VI}]$. Let $\lfloor\cdot\rfloor: \mathbb{R} \rightarrow \mathbb{Z}$ denote the "greatest integer less than or equal to" function.

Proposition D Assume that $W$ is as described immediately above.

(1) The number of reducible PSL $(2, \mathbb{C})$-characters of $\pi_{1}(W)$ is

$$
\left\lfloor\frac{|a q r+b p r+c p q|}{2}\right\rfloor+ \begin{cases}1 & \text { if } \operatorname{gcd}(p, q, r) \text { is odd } \\ 2 & \text { if } \operatorname{gcd}(p, q, r) \text { is even } .\end{cases}
$$

(2) The number of $P S L(2, \mathbb{C})$-characters of $\pi_{1}(W)$ representations with image a dihedral group of order at least 4 is

$$
\sigma(q, r)\left\lfloor\frac{p}{2}\right\rfloor+\sigma(p, r)\left\lfloor\frac{q}{2}\right\rfloor+\sigma(p, q)\left\lfloor\frac{r}{2}\right\rfloor-2 \sigma(p, q) \sigma(p, r) \sigma(q, r)
$$

where $\sigma(m, n)$ is one if $m, n$ are both even, and zero otherwise.

(3) The number of $P S L(2, \mathbb{C})$-characters of $\pi_{1}(W)$ is

$$
\begin{gathered}
\left\lfloor\frac{p}{2}\right\rfloor\left\lfloor\frac{q}{2}\right\rfloor\left\lfloor\frac{r}{2}\right\rfloor+\left\lfloor\frac{p-1}{2}\right\rfloor\left\lfloor\frac{q-1}{2}\right\rfloor\left\lfloor\frac{r-1}{2}\right\rfloor+\left\lfloor\frac{|a q r+b p r+c p q|}{2}\right\rfloor-\left\lfloor\frac{\operatorname{gcd}(p q, p r, q r)}{2}\right\rfloor \\
+\left\lfloor\frac{\operatorname{gcd}(p, q)}{2}\right\rfloor+\left\lfloor\frac{\operatorname{gcd}(p, r)}{2}\right\rfloor+\left\lfloor\frac{\operatorname{gcd}(q, r)}{2}\right\rfloor+1 .
\end{gathered}
$$

It is possible to perform a similar calculation in the case of a Haken small Seifert manifold. The only difference which arises is that in this case the set of reducible characters is positive dimensional, reflecting the fact that such a manifold has infinite first homology (see Remark 3.3).

We prove Theorem A, Corollary B and Theorem C in $\S 1$. It turns out that with applications in mind, it is useful to develop $\operatorname{PSL}(2, \mathbb{C})$ versions of our results. This is done in $\S 2$. Finally in $\S 3$ we prove Propositon D.

\section{Jumps in multiplicities of zeros}

Throughout this section we shall assume that $X_{0} \subset X(M)$ is a non-trivial, irreducible curve and $R_{0}$ is the unique 4-dimensional irreducible subvariety of $R(M)$ for which $t\left(R_{0}\right)=$ $R(M)$. Suppose that $\rho \in R_{0}$ has a non-abelian image and $\rho(\alpha(r)) \in\{ \pm I\}$ but $\rho\left(\pi_{1}(\partial M)\right) \nsubseteq$ 
$\{ \pm I\}$. To simplify the notation, we shall use the symbol $A d \rho$ to designate either the restriction of $A d \rho$ to a subgroup of $\pi_{1}(M)$ or a quotient of a subgroup of $\pi_{1}(M)$ through which $A d \rho$ factors. In particular, as $\rho(\alpha(r)) \in\{ \pm I\}$, Ad $\rho$ factors through $\pi_{1}(M(r))$. We assume that $H^{1}(M(r) ; A d \rho)=0$.

The following result is essential to our analysis. Its proof in the case of an irreducible representation can be found in [6]. The case of a reducible representation is handled in Corollary B of [2].

Theorem 1.1 Suppose that $\rho \in \overline{R^{\text {irr }}(M)}$ is a non-abelian representation for which $\rho(\alpha(r)) \in$ $\{ \pm I\}$ but $\rho\left(\pi_{1}(\partial M)\right)$ is not contained in $\{ \pm I\}$, and $H^{1}(M(r) ; A d \rho)=0$. Then

(1) $\rho$ is a simple point of $R(M)$ and the algebraic component of $R(M)$ which contains it is 4-dimensional.

(2) $\chi_{\rho}$ is a simple point of $\overline{X^{i r r}(M)}$ and the algebraic component of $\overline{X^{i r r}(M)}$ which contains it is a curve.

(3) there is an analytic 2-disk $D$, smoothly embedded in $R(M)$ and transverse to $\mathcal{O}(\rho)$ at $\rho$, such that $t \mid D$ is an analytic isomorphism onto a neighbourhood of $\chi_{\rho}$ in $\overline{X^{i r r}(M)}$.

$\underset{\simeq \simeq \cong}{(4)}$ is scheme reduced and there is a commutative diagram [h=2.5em] $T_{\rho}^{Z a r} \mathcal{O}(\rho) T_{\rho}^{Z a r} R(M)^{d t} T_{\chi_{\rho}}^{Z a r} \overline{X^{i r r}(M)}$ $\cong \cong \cong$

$B^{1}(M ; A d \rho) Z^{1}(M ; A d \rho) H^{1}(M ; A d \rho)$.

\subsection{The proof of Theorem A (1)}

The proof splits naturally into the two cases: $\rho\left(\pi_{1}(\partial M)\right)$ is diagonalisable, $\rho\left(\pi_{1}(\partial M)\right)$ is parabolic. We begin by developing material common to both.

By Theorem 1.1, our hypotheses imply that $\nu(x)=\chi_{\rho}$ is a smooth point of $X_{0}$ and therefore $\nu: X_{0}^{\nu} \rightarrow X_{0}$ provides an analytic isomorphism from a small neighbourhood of $x$ in $X_{0}^{\nu}$ to a small neighbourhood of $\chi_{\rho} \in X_{0}$. In particular the following lemma holds.

Lemma $1.2 Z_{\chi_{\rho}}\left(f_{\gamma}\right)$ is defined and equals $Z_{x}\left(\tilde{f}_{\gamma}\right)$ for each $\gamma \in \pi_{1}(M)$.

Theorem 1.1 also shows that we may choose a smooth, analytic disk $D \subset R_{0}$ such that $D \cap \mathcal{O}(\rho)=\{\rho\}$ and $t \mid D$ is an analytic isomorphism between $D$ and a neighbourhood of $\chi_{\rho}$ in $X(M)$. Let $\sigma:((-\epsilon, \epsilon), 0) \rightarrow\left(t(D), \chi_{\rho}\right)$ be a smooth path with non-zero tangent at 0 . Then $\sigma$ lifts to a smooth deformation $\rho_{s}$ of $\rho=\rho_{0}$. This deformation admits an expansion of the form

$$
\rho_{s}=\exp \left(s u_{1}+s^{2} u_{2}+s^{3} u_{3}+\ldots\right) \rho
$$

where $u_{j}: \pi \rightarrow s l_{2}(\mathbb{C})[10]$. Thus for each $\gamma \in \pi_{1}(M)$,

$$
\rho_{s}(\gamma)=\exp \left(s u_{1}(\gamma)+s^{2} u_{2}(\gamma)+s^{3} u_{3}(\gamma)+\ldots\right) \rho(\gamma) \in S L(2, \mathbb{C})
$$


It is well known (cf. [10]) that $u_{1} \in Z^{1}(M ; A d \rho)$ and that under the isomorphism $T_{\rho}^{Z a r} R(M)$ $\rightarrow Z^{1}(M ; A d \rho)$ (Theorem 1.1), we have $\left.\frac{d}{d s}\right|_{s=0} \rho_{s} \mapsto u_{1}$. Hence if $\bar{u}_{1}$ denotes the class of $u_{1}$ in $H^{1}(M ; A d \rho) \cong \mathbb{C}$ (Theorem 1.1), the fact that $\rho_{s}$ has a non-zero tangent at $s=0$ which is not in $T_{\rho}^{Z a r} \mathcal{O}(\rho)$ implies that $\bar{u}_{1} \neq 0$.

Lemma 1.3 (1) If $\gamma \in \pi_{1}(M)$, then $f_{\gamma}\left(\chi_{\rho_{s}}\right)$ has a Taylor expansion of the form

$$
\begin{aligned}
f_{\gamma}\left(\chi_{\rho_{s}}\right)= & f_{\gamma}\left(\chi_{\rho}\right)+2 I_{\gamma}\left(\chi_{\rho}\right) \operatorname{tr}\left(u_{1}(\gamma) \rho(\gamma)\right) s+\left\{2 I_{\gamma}\left(\chi_{\rho}\right) \operatorname{tr}\left(u_{2}(\gamma) \rho(\gamma)\right)\right. \\
& \left.+\operatorname{tr}\left(u_{1}(\gamma) \rho(\gamma)\right)^{2}+I_{\gamma}\left(\chi_{\rho}\right) \operatorname{tr}\left(u_{1}(\gamma)^{2} \rho(\gamma)\right)\right\} s^{2}+O\left(s^{3}\right) .
\end{aligned}
$$

(2) If $\gamma \in \pi_{1}(M)$ and $0=u_{1}(\gamma)=u_{2}(\gamma)=\ldots=u_{k-1}(\gamma)$ where $k>1$, then $f_{\gamma}\left(\chi_{\rho_{s}}\right)$ has a Taylor expansion of the form

$$
\begin{aligned}
f_{\gamma}\left(\chi_{\rho_{s}}\right)= & f_{\gamma}\left(\chi_{\rho}\right)+2 I_{\gamma}\left(\chi_{\rho}\right) \operatorname{tr}\left(u_{k}(\gamma) \rho(\gamma)\right) s^{k}+2 I_{\gamma}\left(\chi_{\rho}\right) \operatorname{tr}\left(u_{k+1}(\gamma) \rho(\gamma)\right) s^{k+1} \\
& +O\left(s^{k+2}\right) .
\end{aligned}
$$

Proof. Just develop $f_{\gamma}\left(\chi_{\rho_{s}}\right)=\left\{\operatorname{tr}\left(\rho_{s}(\gamma)\right)\right\}^{2}-4$ using the expansion $\exp (A)=\sum_{j=1}^{\infty} \frac{A^{j}}{j !}$ for $A \in s l_{2}(\mathbb{C})$.

Our analysis now divides into two cases depending on whether $\rho\left(\pi_{1}(\partial M)\right)$ is a diagonalisable or parabolic.

\subsubsection{The case when $\rho\left(\pi_{1}(\partial M)\right)$ is diagonalisable.}

Since $\rho(\beta)$ is diagonalisable, but not $\pm I$, it follows that $f_{\beta}\left(\chi_{\rho}\right) \neq 0$, and thus

$$
Z_{x}\left(\tilde{f}_{\beta}\right)=0
$$

On the other hand since $\rho(\alpha(r))= \pm I$, Lemma 1.3 implies that

$$
f_{\alpha(r)}\left(\chi_{\rho_{s}}\right)=2 \operatorname{tr}\left(u_{1}(\alpha(r))^{2}\right) s^{2}+O\left(s^{3}\right) .
$$

Thus $Z_{x}\left(\tilde{f}_{\alpha(r)}\right) \geq 2=Z_{x}\left(\tilde{f}_{\beta}\right)+2$ (cf. Lemma 1.2) and so this case of the theorem is complete.

\subsubsection{The case when $\rho\left(\pi_{1}(\partial M)\right)$ is parabolic.}

As we have assumed that $\rho(\beta) \neq \pm I$, we may suppose, after possibly replacing $\rho$ by a conjugate representation, that

$$
\rho(\beta)= \pm\left(\begin{array}{ll}
1 & 1 \\
0 & 1
\end{array}\right)
$$


Evidently $f_{\beta}\left(\chi_{\rho}\right)=0$, so that $Z_{x}\left(\tilde{f}_{\beta}\right) \geq 1$. If $f_{\beta} \mid X_{0}=0$, then by [9, Proposition 1.5.4] there is a neighbourhood $U$ of $\rho$ in $R_{0}$ such that $\rho^{\prime}(\beta)$ is parabolic for each $\rho^{\prime} \in U$. Hence the fact that $\alpha(r)$ and $\beta$ commute implies that $\rho^{\prime}(\alpha(r))$ is either parabolic or central in $S L(2, \mathbb{C})$ for each such $\rho^{\prime}$. It follows that $f_{\alpha(r)} \mid X_{0}=0$ and hence that the inequality of part (1) of Theorem A holds. We shall therefore assume below that $f_{\beta} \mid X_{0} \neq 0$ and we take

$$
k_{0}=Z_{x}\left(\tilde{f}_{\beta}\right)<\infty
$$

Our first task is to replace the curve

$$
\rho_{s}=\exp \left(s u_{1}+s^{2} u_{2}+s^{3} u_{3}+\ldots\right) \rho
$$

described above by a new deformation of $\rho$ which is better adapted to the calculation we have in hand.

Lemma 1.4 The equality $Z_{x}\left(\tilde{f}_{\beta}\right) \geq k$ holds if and only if there exists a smooth curve $\phi_{s} \in R(M)$ of the form $\phi_{s}=\exp \left(s v_{1}+s^{2} v_{2}+s^{3} v_{3}+\ldots\right) \rho$ where

(i) $\bar{v}_{1}=\bar{u}_{1} \in H^{1}(M ; A d \rho)$.

(ii) $v_{i}(\beta)=0$ for $1 \leq i<k$.

(iii) $v_{k}(\beta)=\left(\begin{array}{ll}0 & 0 \\ c & 0\end{array}\right)$ for some $c \in \mathbb{C}$.

Moreover, $Z_{x}\left(\tilde{f}_{\beta}\right)=k$ if and only if there exists a curve of this type with $c \neq 0$.

Proof. First assume that there is a smooth curve in $R(M)$ of the form $\phi_{s}=\exp \left(s v_{1}+\right.$ $\left.s^{2} v_{2}+\ldots\right) \rho$ which satisfies the three conditions of the hypotheses. Then Lemma 1.3 implies that

$$
f_{\beta}\left(\chi_{\phi_{s}}\right)=4 c s^{k}+O\left(s^{k+1}\right) .
$$

Thus $Z_{x}\left(\tilde{f}_{\beta}\right) \geq k$ with equality if and only if $c \neq 0$.

Conversely assume that $Z_{x}\left(\tilde{f}_{\beta}\right) \geq k$. We shall alter the deformation $\rho_{s}=\exp \left(s u_{1}+\right.$ $\left.s^{2} u_{2}+s^{3} u_{3}+\ldots\right) \rho$ inductively to show that there is a new deformation $\phi_{s}$ of the required form.

For each $A \in \operatorname{sl}_{2}(\mathbb{C})$, let $u_{A} \in B^{1}(M ; A d \rho)$ be the 1-coboundary defined by

$$
u_{A}(\gamma)=A-A d \rho(\gamma)(A)
$$

Suppose that $u_{1}(\beta)=\left(\begin{array}{cc}a & b \\ c & -a\end{array}\right)$ and set $B=\left(\begin{array}{cc}-\frac{1}{2}(a+b) & 0 \\ a & \frac{1}{2}(a+b)\end{array}\right)$. Standard identities involving the exponential map of a Lie group imply that the curve of representations $\rho_{s}^{\prime}=\exp (s B) \rho_{s} \exp (-s B)$ admits an expansion of the form

$$
\rho_{s}^{\prime}=\exp \left(s v_{1}+s^{2} u_{2}^{\prime}+\cdots\right) \rho
$$


where $v_{1}=u_{1}+u_{B}$, so $\bar{v}_{1}=\bar{u}_{1}$. Observe that $v_{1}(\beta)=\left(u_{1}+u_{B}\right)(\beta)=\left(\begin{array}{ll}0 & 0 \\ c & 0\end{array}\right)$ and so by the first paragraph of the proof, $Z_{x}\left(\tilde{f}_{\beta}\right) \geq 1$ with equality if and only if $c \neq 0$. This completes the case where $k=1$.

Assume inductively that we can find such a curve whenever $Z_{x}\left(\tilde{f}_{\beta}\right) \geq n \geq 1$ and suppose that in fact $Z_{x}\left(\tilde{f}_{\beta}\right) \geq n+1$. Then there is a smooth curve in $R(M)$ of the form $\phi_{s}=\exp \left(s v_{1}+s^{2} v_{2}+\ldots\right) \rho$ which satisfies the three conditions of the hypotheses with $k=n$. By the first paragraph of the proof $c=0$, that is $v_{n}(\beta)=0$. Write $v_{n+1}(\beta)=\left(\begin{array}{cc}a & b \\ c & -a\end{array}\right)$ and set $B=\left(\begin{array}{cc}-\frac{1}{2}(a+b) & 0 \\ a & \frac{1}{2}(a+b)\end{array}\right)$. The curve $\phi_{s}^{\prime}=\exp \left(s^{n+1} B\right) \phi_{s} \exp \left(-s^{n+1} B\right)$ may be expanded as

$$
\phi_{s}^{\prime}=\exp \left(s v_{1}+\cdots+s^{n} v_{n}+s^{n+1} v_{n+1}^{\prime}+s^{n+2} v_{n+2}^{\prime}+\cdots\right) \rho
$$

where $v_{n+1}^{\prime}=v_{n+1}+u_{B}$. Clearly $v_{n+1}^{\prime}(\beta)=\left(\begin{array}{ll}0 & 0 \\ c & 0\end{array}\right)$. Thus the first paragraph of the proof shows that $Z_{x}\left(\tilde{f}_{\beta}\right) \geq n+1$ with equality if and only if $c \neq 0$. This completes the induction.

In what follows we suppose that the curve of representations $\rho_{s}$ admits an expansion of the form described in Lemma 1.4:

$$
\rho_{s}=\exp \left(s u_{1}+s^{2} u_{2}+s^{3} u_{3}+\ldots+s^{k} u_{k}+O\left(s^{k+1}\right)\right) \rho
$$

where

$$
u_{i}(\beta)=0 \text { for all } i \text { such that } 1 \leq i<k_{0}=Z_{x}\left(\tilde{f}_{\beta}\right)
$$

and

$$
u_{k_{0}}(\beta)=\left(\begin{array}{cc}
0 & 0 \\
c & 0
\end{array}\right)
$$

for some $c \in \mathbb{C}^{*}=\mathbb{C} \backslash\{0\}$.

Lemma 1.5 Given the restrictions we have imposed on the expansion of $\rho_{s}$, the following identities hold.

(1) $u_{i}(\alpha(r))=\left(\begin{array}{cc}0 & y_{i} \\ 0 & 0\end{array}\right)$ for some $y_{i} \in \mathbb{C}, 1 \leq i \leq k_{0}$.

(2) $u_{k_{0}+1}(\alpha(r))=\left(\begin{array}{cc}-\frac{1}{2} y_{1} c & y_{k_{0}+1} \\ y_{1} c & \frac{1}{2} y_{1} c\end{array}\right)$ for some $y_{k_{0}+1} \in \mathbb{C}$. 
Proof. The fact that each $\rho_{s}$ is a homomorphism forces certain relations to hold between the functions $u_{i}$. In particular for all $1 \leq i \leq k_{0}$,

$$
\begin{gathered}
u_{i}(\alpha(r) \beta)=u_{i}(\alpha(r))+u_{i}(\beta)+\frac{1}{2} \sum_{j=1}^{k_{0}-1}\left(u_{j} \smile u_{k_{0}-j}\right)(\alpha(r), \beta) \\
+\sum_{l=1}^{i} A_{l}\left(u_{1}, \cdots, u_{i-2}\right)(\alpha(r), \beta)
\end{gathered}
$$

where $A_{l}\left(u_{1}, \cdots, u_{i-2}\right)(\alpha(r), \beta)$ is a sum of monomials in Lie brackets between the $u_{j}(\alpha(r))$ and $\operatorname{Ad} \rho(\alpha(r))\left(u_{j^{\prime}}(\beta)\right)$ with $1 \leq j, j^{\prime} \leq i-2$ (see [10] and [1]). As $u_{j}(\beta)=0$ for $1 \leq j<k_{0}$ we have

$$
u_{i}(\alpha(r) \beta)=u_{i}(\alpha(r))+u_{i}(\beta) \text { for } 1 \leq i \leq k_{0} .
$$

Similarly for this range of indices we have

$$
u_{i}(\beta \alpha(r))=u_{i}(\beta)+A d \rho(\beta)\left(u_{i}(\alpha(r))\right) .
$$

Since $\beta$ and $\alpha(r)$ commute in $\pi_{1}(\partial M), u_{i}(\alpha(r) \beta)=u_{i}(\beta \alpha(r))$ and hence the calculations above show

$$
u_{i}(\alpha(r))=A d \rho(\beta)\left(u_{i}(\alpha(r))\right) \text { for } 1 \leq i \leq k_{0} .
$$

Since $\rho(\beta)= \pm\left(\begin{array}{ll}1 & 1 \\ 0 & 1\end{array}\right)$, if we set $u_{i}(\alpha(r))=\left(\begin{array}{cc}x_{i} & y_{i} \\ z_{i} & -x_{i}\end{array}\right)$, then these conditions imply that $x_{i}=z_{i}=0$ for $1 \leq i \leq k_{0}$. Thus for these values of $i$ we obtain $u_{i}(\alpha(r))=$ $\left(\begin{array}{cc}0 & y_{i} \\ 0 & 0\end{array}\right), y_{i} \in \mathbb{C}$, and therefore part (1) of the lemma holds.

Next we consider part (2). As above we have identities

$$
\begin{aligned}
& u_{k_{0}+1}(\alpha(r) \beta)=u_{k_{0}+1}(\alpha(r))+u_{k_{0}+1}(\beta)+\frac{1}{2}\left[u_{1}(\alpha(r)), u_{k_{0}}(\beta)\right] \\
& u_{k_{0}+1}(\beta \alpha(r))=u_{k_{0}+1}(\beta)+A d \rho(\beta)\left(u_{k_{0}+1}(\alpha(r))\right)+\frac{1}{2}\left[u_{k_{0}}(\beta), A d \rho(\beta)\left(u_{1}(\alpha(r))\right)\right],
\end{aligned}
$$

where $[\cdot, \cdot]$ denotes the Lie bracket on $s l_{2}(\mathbb{C})$. Now $\rho(\beta)= \pm\left(\begin{array}{ll}1 & 1 \\ 0 & 1\end{array}\right)$ commutes with $u_{1}(\alpha(r))=\left(\begin{array}{cc}0 & y_{1} \\ 0 & 0\end{array}\right)$ and $u_{k_{0}+1}(\alpha(r) \beta)=u_{k_{0}+1}(\beta \alpha(r))$, so we obtain
$u_{k_{0}+1}(\alpha(r))=A d \rho(\beta)\left(u_{k_{0}+1}(\alpha(r))\right)-\left[u_{1}(\alpha(r)), u_{k_{0}}(\beta)\right]$.

Thus

$$
\left(\begin{array}{cc}
x_{k_{0}+1} & y_{k_{0}+1} \\
z_{k_{0}+1} & -x_{k_{0}+1}
\end{array}\right)=\left(\begin{array}{cc}
x_{k_{0}+1}+z_{k_{0}+1}-y_{1} c & y_{k_{0}+1}-2 x_{k_{0}+1}-z_{k_{0}+1} \\
z_{k_{0}+1} & -\left(x_{k_{0}+1}+z_{k_{0}+1}-y_{1} c\right)
\end{array}\right)
$$


and therefore $u_{k_{0}+1}(\alpha(r))=\left(\begin{array}{cc}-\frac{1}{2} y_{1} c & y_{k_{0}+1} \\ y_{1} c & \frac{1}{2} y_{1} c\end{array}\right)$, as claimed.

Consider the expansion

$$
\rho_{s}(\alpha(r))=\exp \left(s u_{1}(\alpha(r))+\cdots+s^{k_{0}+2} u_{k_{0}+2}(\alpha(r))+O\left(s^{k_{0}+3}\right)\right) \rho(\alpha(r))
$$

and denote by $A_{s}$ the sum $s u_{1}(\alpha(r))+\cdots+s^{k_{0}+2} u_{k_{0}+2}(\alpha(r))$. Then

$$
\operatorname{tr}\left(\chi_{\rho_{s}}(\alpha(r))\right)= \pm\left(2+\operatorname{tr}\left(A_{s}\right)+\frac{1}{2 !} \operatorname{tr}\left(A_{s}^{2}\right)+\ldots+\frac{1}{\left(k_{0}+2\right) !} \operatorname{tr}\left(A_{s}^{k_{0}+2}\right)+O\left(s^{k_{0}+3}\right)\right) .
$$

Lemma 1.5 implies that $u_{i}(\alpha(r)) u_{j}(\alpha(r))=0$ for all $1 \leq i, j \leq k_{0}$. So, by use of the multinomial formula and Lemma 1.5, we obtain

$$
\operatorname{tr}\left(A_{s}^{2}\right)=s^{k_{0}+2} \operatorname{tr}\left(u_{1}(\alpha(r)) u_{k+1}(\alpha(r))\right)+O\left(s^{k_{0}+3}\right)=y_{1}^{2} c s^{k_{0}+2}+O\left(s^{k_{0}+3}\right)
$$

and

$$
\operatorname{tr}\left(A_{s}^{i}\right)=O\left(s^{k_{0}+3}\right) \text { for } 1 \leq i \leq k_{0}+2 \text { and } i \neq 2 .
$$

This implies that

$$
\operatorname{tr}\left(\chi_{\rho_{s}}(\alpha(r))\right)= \pm\left(2+\left(\frac{y_{1}^{2} c}{2}\right) s^{k_{0}+2}+O\left(s^{k_{0}+3}\right)\right)
$$

and hence

$$
f_{\alpha(r)}\left(\chi_{\rho_{s}}(\alpha(r))\right)=\operatorname{tr}\left(\chi_{\rho_{s}}(\alpha(r))\right)^{2}-4=2 y_{1}^{2} c s^{k_{0}+2}+O\left(s^{k_{0}+3}\right) .
$$

Thus $Z_{x}\left(\tilde{f}_{\alpha(r)}\right) \geq k_{0}+2=Z_{x}\left(\tilde{f}_{\beta}\right)+2$. This completes the proof of part (1) of Theorem A.

\subsection{The proof of Theorem A (2)}

Again our analysis splits into the two cases $\rho\left(\pi_{1}(\partial M)\right)$ diagonalisable and $\rho\left(\pi_{1}(\partial M)\right)$ parabolic. We begin, as before, developing material common to both.

According to the hypotheses of this part of Theorem A, $f_{\beta} \mid X_{0} \neq 0$. We claim that the condition $H^{1}(M(r) ; A d \rho)=0$ implies that $f_{\alpha(r)} \mid X_{0} \neq 0$ as well. Otherwise $\rho^{\prime}(\alpha(r))$ is either parabolic or lies in $\{ \pm I\}$ for each $\rho^{\prime} \in R_{0}$. If the former occurs for some $\rho^{\prime}$, then it holds for $\rho^{\prime}$ in a Zariski open subset of $R_{0}[9$, Proposition 1.5.4] and hence the fact that $\alpha(r)$ and $\beta$ commute implies that $f_{\beta} \mid X_{0}=0$, contrary to our assumptions. Thus $\rho^{\prime}(\alpha(r))=\epsilon I$ for all $\rho^{\prime} \in R_{0}$ and some fixed $\epsilon \in\{ \pm 1\}$. Let $\bar{R}(M)$ denote the $P S L(2, \mathbb{C})$-representation variety of $M$ [5] and denote by $\bar{R}_{0}$ the image of $R_{0}$ in $\bar{R}(M)$ under the finite-to-one map $R(M) \rightarrow$ $\bar{R}(M)$. Clearly $\bar{R}_{0}$ is 4-dimensional and is contained in $\bar{R}(M(r)) \subset \bar{R}(M)$. In particular, $4 \leq \operatorname{dim}_{\rho} \bar{R}(M(r)) \leq \operatorname{dim} Z^{1}(M(r) ; A d \rho)$. But this is impossible as our assumption that 
$H^{1}(M(r) ; A d \rho)=0$ implies that $Z^{1}(M(r) ; A d \rho) \cong \mathbb{C}^{3}$. Hence $f_{\alpha(r)} \mid X_{0} \neq 0$. In particular we see that

$$
Z_{x}\left(\tilde{f}_{\alpha(r)}\right)<\infty .
$$

Next we show that $\rho(\beta)$ cannot be $\pm I$. First we need a lemma.

Lemma 1.6 Let $\Gamma$ be a finitely generated group, $\gamma \in \Gamma$, and $X_{0} \subset X(\Gamma)$ a curve. Then for each $n \in \mathbb{Z}$, there is some $g_{n} \in \mathbb{C}\left(X_{0}\right)$ such that $f_{\gamma^{n}}(x)=g_{n}(x) f_{\gamma}(x)$ for each $x \in X_{0}$. Furthermore if $\rho \in R_{0}$, then $g_{n}\left(\chi_{\rho}\right)=0$ if and only if $\rho(\gamma)$ has finite order $d>2$ where $d$ divides $2 n$.

Proof. Let $R_{0}$ be the unique 4-dimensional subvariety of $R(M)$ for which $t\left(R_{0}\right)=X_{0}$. There is a finite extension $F$ of $\mathbb{C}\left(R_{0}\right)$ and a tautological representation $P: \pi \rightarrow P S L_{2}(F)$ defined by

$$
P(\zeta)=\left(\begin{array}{ll}
a & b \\
c & d
\end{array}\right)
$$

where the functions $a, b, c$ and $d$ satisfy the identity

$$
\rho(\gamma)=\left(\begin{array}{ll}
a(\rho) & b(\rho) \\
c(\rho) & d(\rho)
\end{array}\right)
$$

for all $\rho \in R_{0}([8])$. In particular, for each $\rho \in R_{0}$ we have $f_{\zeta}\left(\chi_{\rho}\right)=\operatorname{tr}(P(\zeta))(\rho)^{2}-4$. By passing to an extension field of $F$ if necessary, we may assume that $P(\gamma)$ is an uppertriangular matrix, say $P(\gamma)=\left(\begin{array}{cc}a & * \\ 0 & a^{-1}\end{array}\right)$. Then for $n \in \mathbb{Z}$,

$$
f_{\gamma^{n}}=\left(a^{n}-a^{-n}\right)^{2}=\left(a-a^{-1}\right)^{2}\left(a^{(|n|-1)}+a^{(|n|-3)}+\ldots+a^{-(|n|-1)}\right)^{2} .
$$

If we set $g_{n}=a^{2(1-n)}\left(\frac{a^{2 n}-1}{a^{2}-1}\right)=\left(a^{(|n|-1)}+a^{(|n|-3)}+\ldots+a^{-(|n|-1)}\right)^{2}$, then $f_{\gamma^{n}}=g_{n} f_{\gamma}$. Further $g_{n}\left(\chi_{\rho}\right)=0$ if and only if $a(\rho)^{2} \neq 1$ and $a(\rho)^{2 n}=1$, which is what we set out to prove.

Corollary 1.7 Given that $Z_{x}\left(\tilde{f}_{\beta}\right)<Z_{x}\left(\tilde{f}_{\alpha(r)}\right)<\infty$, then $\rho(\beta) \neq \pm I$.

Proof. Since $\rho(\alpha(r))= \pm I$ while $\rho\left(\pi_{1}(\partial M)\right) \nsubseteq\{ \pm I\}$, it suffices to show that $\beta \neq n \alpha(r)$ for any $n \in \mathbb{Z}$.

Suppose to the contrary that $\beta=n \alpha(r)$ for some $n$. Since $Z_{x}\left(\tilde{f}_{\beta}\right)<\infty$, we have $n \neq 0$. According to Lemma 1.6, $\tilde{f}_{\beta}=\tilde{f}_{n \alpha}=\tilde{g}_{n} \tilde{f}_{\alpha}$ where $\tilde{g}_{n}(x)=n^{2} \neq 0$. Hence

$$
Z_{x}\left(\tilde{f}_{\beta}\right)=Z_{x}\left(\tilde{f}_{n \alpha(r)}\right)=Z_{x}\left(\tilde{f}_{\alpha}\right)+Z_{x}\left(\tilde{g}_{n}\right)=Z_{x}\left(\tilde{f}_{\alpha}\right),
$$


contrary to our assumptions. Thus $\beta$ cannot be a multiple of $\alpha(r)$, and we are done.

We can therefore apply the work of $\S 1.1$ in this section. In particular

$$
Z_{x}\left(\tilde{f}_{\alpha(r)}\right) \geq Z_{x}\left(\tilde{f}_{\beta}\right)+2
$$

The key to showing that equality holds rests in our next lemma.

Lemma 1.8 If $\bar{u}$ is a non-zero class in $H^{1}(M ; A d \rho) \cong \mathbb{C}$, then $u(\alpha(r)) \neq 0$.

Proof. Denote by $V(r)$ the surgery torus in $M(r)=M \cup V(r)$. If $j: \partial M \rightarrow V(r)$ is the inclusion, let $j_{\#}: \pi_{1}(\partial M) \rightarrow \pi_{1}(V(r))$ and $j^{*}: H^{1}(V(r) ; A d \rho) \rightarrow H^{1}(\partial M ; A d \rho)$ denote the associated homomorphisms.

Suppose that $u(\alpha(r))=0$ and let $u_{0} \in Z^{1}(\partial M ; A d \rho)$ be the composition $\pi_{1}(\partial M) \rightarrow$ $\pi_{1}(M) \stackrel{u}{\longrightarrow} \mathbb{C}$. An easy calculation shows that $u_{0}\left(\alpha(r)^{n}\right)=0$ for each $n \in \mathbb{Z}$, and thus $u_{0}^{\prime}=u_{0} \circ j_{\#}^{-1}: \pi_{1}(V(r)) \rightarrow s l_{2}(\mathbb{C})$ is a well-defined 1-cocycle on $\pi_{1}(V(r))$. Clearly $u_{0}=u_{0}^{\prime} \circ j_{\#}$ so that $\bar{u}_{0}=j^{*}\left(\overline{u_{0}^{\prime}}\right)$. The Mayer-Vietoris sequence for cohomology with twisted coefficients associated to the decomposition $M(r)=M \cup_{\partial M} V(r)$ shows that $\bar{u}$ is in the image of the natural homomorphism $H^{1}(M(r) ; A d \rho) \rightarrow H^{1}(M ; A d \rho)$. Since $H^{1}(M(r) ; A d \rho)=0$, this implies that $\bar{u}=0$, contrary to our assumptions. Thus $u(\alpha(r)) \neq 0$.

\subsubsection{The case when $\rho\left(\pi_{1}(\partial M)\right)$ is diagonalisable.}

Recall from $\S 1.1 .1$, that $Z_{x}\left(\tilde{f}_{\beta}\right)=0$ while

$$
f_{\alpha(r)}\left(\chi_{\rho_{s}}\right)=2 \operatorname{tr}\left(u_{1}(\alpha(r))^{2}\right) s^{2}+O\left(s^{3}\right)
$$

where $u_{1} \in Z^{1}(M ; A d \rho)$ is non-zero cohomologically. The desired result is then a consequence of

Lemma 1.9 If $\bar{u}$ is a non-zero class in $H^{1}(M ; A d \rho)$, then $\operatorname{tr}\left(u(\alpha(r))^{2}\right) \neq 0$.

Proof. Without loss of generality we may suppose that $\rho\left(\pi_{1}(\partial M)\right) \subset D$, the group of diagonal matrices in $S L(2, \mathbb{C})$. We claim that $u(\alpha(r))$ is also a diagonal matrix. To see this, first observe that by our hypotheses on $\rho, \rho(\beta)=\left(\begin{array}{cc}\lambda & 0 \\ 0 & \lambda^{-1}\end{array}\right)$, where $\lambda \neq \pm 1$. Now

$$
u(\alpha(r))+u(\beta)=u(\alpha(r) \beta)=u(\beta \alpha(r))=u(\beta)+\left(\begin{array}{cc}
\lambda & 0 \\
0 & \lambda^{-1}
\end{array}\right) u(\alpha(r))\left(\begin{array}{cc}
\lambda^{-1} & 0 \\
0 & \lambda
\end{array}\right)
$$


and hence, $u(\alpha(r))=\left(\begin{array}{cc}\lambda & 0 \\ 0 & \lambda^{-1}\end{array}\right) u(\alpha(r))\left(\begin{array}{cc}\lambda^{-1} & 0 \\ 0 & \lambda\end{array}\right)$. As $\lambda^{2} \neq 1$, this can occur only if $u(\alpha(r))$ is diagonal.

We may now write $u(\alpha(r))=z\left(\begin{array}{cc}1 & 0 \\ 0 & -1\end{array}\right)$ for some $z \in \mathbb{C}$. Note that $z \neq 0$ by Lemma 1.8. Then $\operatorname{tr}\left(u(r)^{2}\right)=2 z^{2} \neq 0$, and the lemma is proved.

\subsubsection{The case when $\rho\left(\pi_{1}(\partial M)\right)$ is parabolic.}

The hypotheses of part (2) preclude the possibility that $f_{\beta} \mid X_{0}=0$, and so we shall take

$$
Z_{x}\left(\tilde{f}_{\beta}\right)=k_{0}<\infty
$$

According to $\S 1.1 .2$, there is a smooth deformation of $\rho$ :

$$
\rho_{s}=\exp \left(s u_{1}+s^{2} u_{2}+s^{3} u_{3}+\ldots+s^{k} u_{k}+O\left(s^{k+1}\right)\right) \rho
$$

where $u_{i}(\beta)=0$ for all $i$ such that $1 \leq i<k_{0}$ and $u_{k_{0}}(\beta)=\left(\begin{array}{ll}0 & 0 \\ c & 0\end{array}\right)$ for some $c \in \mathbb{C}^{*}$. Furthermore, the local development of $\tilde{f}_{\alpha(r)}$ with respect to the deformation $\chi_{\rho_{s}}$ of $\chi_{\rho}$ is

$$
f_{\alpha(r)}\left(\chi_{\rho_{s}}(\alpha(r))\right)=2 y_{1}^{2} c s^{k_{0}+2}+O\left(s^{k_{0}+3}\right)
$$

where $y_{1} \in \mathbb{C}$ is determined by $u_{1}(\alpha(r))=\left(\begin{array}{cc}0 & y_{1} \\ 0 & 0\end{array}\right)$. According to Lemma 1.8, $y_{1} \neq 0$ and therefore

$$
Z_{x}\left(\tilde{f}_{\alpha(r)}\right)=k_{0}+2=Z_{x}\left(\tilde{f}_{\beta}\right)+2 .
$$

This completes the proof of Theorem A.

\subsection{The proof of Corollary B}

Assume that $f_{\beta} \mid X_{0}=0$. If $\beta \in H_{1}(\partial M) \backslash\{n \alpha(r) \mid n \in \mathbb{Z} \backslash\{0\}\}$, then $\rho(\beta) \neq \pm I$ and so part (1) of Theorem A implies that $Z_{x}\left(\tilde{f}_{\alpha(r)}\right) \geq Z_{x}\left(\tilde{f}_{\beta}\right)+2=\infty$. Hence $f_{\alpha(r)}=0$. On the other hand if $\beta=n \alpha(r)$ for some non-zero $n \in \mathbb{Z}$, then Lemma 1.6 implies that there is a regular function $g: X_{0} \rightarrow \mathbb{C}$ such that $0=f_{\beta}\left|X_{0}=g f_{\alpha(r)}\right| X_{0}$ and further $g\left(\chi_{\rho^{\prime}}\right)=0$ if and only if $\rho^{\prime}(\alpha(r))$ has finite order $d>2$ where $d$ divides $2 n$. Since $\rho(\alpha(r))= \pm I$, it follows that $g \neq 0$ and thus $f_{\alpha(r)} \mid X_{0}=0$. This proves part (1) of the corollary.

Next assume that $f_{\alpha(r)} \mid X_{0} \neq 0$ and therefore $Z_{x}\left(\tilde{f}_{\alpha(r)}\right)<\infty$. If $\beta \in H_{1}(\partial M) \backslash$ $\{n \alpha(r) \mid n \in \mathbb{Z}\}$ then $\rho(\beta) \neq \pm I$ so that Theorem A (1) gives $Z_{x}\left(\tilde{f}_{\alpha(r)}\right) \geq Z_{x}\left(\tilde{f}_{\beta}\right)+2>$ $Z_{x}\left(\tilde{f}_{\beta}\right)$. Hence by part (2) of that theorem we have $Z_{x}\left(\tilde{f}_{\alpha(r)}\right)=Z_{x}\left(\tilde{f}_{\beta}\right)+2$. If $\beta=n \alpha(r)$ 
for some non-zero $n \in \mathbb{Z}$, then by Lemma 1.6, there is a smooth function $\tilde{g}: \tilde{X}_{0} \rightarrow \mathbb{C}$ such that $\tilde{f}_{\beta}=\tilde{g} \tilde{f}_{\alpha(r)}$ and $\tilde{g}(x) \neq 0$. Then $Z_{x}\left(\tilde{f}_{\beta}\right)=Z_{x}\left(\tilde{g} \tilde{f}_{\alpha(r)}\right)=Z_{x}\left(\tilde{f}_{\alpha(r)}\right)$. Finally $Z_{x}\left(\tilde{f}_{\beta}\right)=\infty$ when $\beta=0$ by convention. This completes the proof of the corollary.

\subsection{The proof of Theorem $\mathrm{C}$}

Assume the hypotheses of Theorem $\mathrm{C}$ and denote by $R_{0}$ the unique irreducible 4-dimensional algebraic subset of $R(M)$ for which $t\left(R_{0}\right)=X_{0}$. Let

- $J \subset \tilde{X}_{0}$ be the set of points $x$ in $\tilde{X}_{0}$ such that $Z_{x}\left(\tilde{f}_{\alpha(r)}\right)>Z_{x}\left(\tilde{f}_{\beta}\right)$ for some $\beta \in \pi_{1}(\partial M)$.

- $K$ be the set of characters in $X_{0}$ of non-abelian $\rho \in R_{0}$ for which $\rho(\alpha(r)) \in\{ \pm I\}$.

By hypothesis, $M(r)$ contains no closed essential surfaces and hence, as $r$ is not a boundary slope, $Z_{x}\left(\tilde{f}_{\alpha(r)}\right) \leq Z_{x}\left(\tilde{f}_{\beta}\right)$ for all ideal points $x$ of $\tilde{X}_{0}$ and $\beta \in H_{1}(\partial M)$ [8, 11.6$]$. Thus $J \subset X_{0}^{\nu}$. We prove next that $\nu: X_{0}^{\nu} \rightarrow X_{0}$ induces a bijection between $J$ and $K$.

Suppose that $x \in J$ and let $\beta \in \pi_{1}(\partial M)$ be such that $Z_{x}\left(\tilde{f}_{\alpha(r)}\right)>Z_{x}\left(\tilde{f}_{\beta}\right)$. Since $M(r)$ is an irreducible, non-Haken small Seifert manifold, $b_{1}(M(r))=0$ and thus $b_{1}(M)=1$. Then [2, Proposition 2.8] implies that $\nu(x) \in X_{0}$ is a non-trivial character and further that there is a non-abelian $\rho \in R_{0}$ such that $\nu(x)=\chi_{\rho}$. Since $Z_{x}\left(\tilde{f}_{\alpha(r)}\right)>Z_{x}\left(\tilde{f}_{\beta}\right)$, we have $\rho(\alpha(r)) \in\{ \pm I\}([8, \S 1.5])$. Thus $\nu(x) \in K$. Note as well that for the induced representation $A d \rho: \pi_{1}(M(r)) \rightarrow P S L(2, \mathbb{C})$ we have $H^{1}(M(r) ; A d \rho)=0$ by the proposition of the introduction. Now $\rho\left(\pi_{1}(\partial M)\right) \not \subset\{ \pm I\}$, as otherwise $\rho$ induces a representation $\pi_{1}(W) \rightarrow$ $\operatorname{PSL}(2, \mathbb{C})$ which is non-diagonalisable, a possibility which contradicts our hypotheses. Thus by $\left[2\right.$, Theorem A] it follows that $\nu(x)$ is a smooth point of $X_{0}$ and therefore $\nu^{-1}(\nu(x))=x$, i.e. $\nu \mid: J \rightarrow K$ is injective.

To prove that $\nu \mid J$ is surjective, suppose that $\chi_{\rho} \in K$ where $\rho \in R_{0}$ is non-abelian and $\rho(\alpha(r))= \pm I$. According to the proposition of the introduction we have $H^{1}(M(r) ; A d \rho)=$ 0 . Note also that $\rho\left(\pi_{1}(\partial M)\right) \not \subset\{ \pm I\}$, as otherwise $\rho$ would induce a non-diagonalisable representation $\pi_{1}(W) \rightarrow P S L(2, \mathbb{C})$. Hence there is some $\beta \in \pi_{1}(\partial M)$ for which $\rho(\beta) \neq \pm I$. If $x \in \nu^{-1}\left(\chi_{\rho}\right)$, then by Theorem A (1) we have $Z_{x}\left(\tilde{f}_{\alpha(r)}\right)>Z_{x}\left(\tilde{f}_{\beta}\right)$. Thus $x \in J$ and hence $\nu(J)=K$. We note also that by part (2) of that theorem

$$
Z_{x}\left(\tilde{f}_{\alpha(r)}\right)-Z_{x}\left(\tilde{f}_{\beta}\right)=2 \quad \text { for each } x \in J
$$

We are now in a position to complete the proof. Since $f_{\alpha(r)} \mid X_{0}$ is non-constant, the function $\tilde{f}_{\alpha(r)}: \tilde{X}_{0} \rightarrow \mathbb{C} P^{1}$ has only finitely many zeros. Hence $Z_{x}\left(\tilde{f}_{\alpha(r)}\right)=0$ for all but finitely many $x \in \tilde{X}_{0}$. In particular

$$
m_{0}=\sum_{x \in \tilde{X}_{0}} \min \left\{Z_{x}\left(\tilde{f}_{\beta}\right) \mid \beta \in H_{1}(\partial M)\right\}<\infty .
$$


We observed above that $Z_{x}\left(\tilde{f}_{\alpha(r)}\right) \leq Z_{x}\left(\tilde{f}_{\beta}\right)$ for all ideal points $x$ of $\tilde{X}_{0}$ and $\beta \in H_{1}(\partial M)$ $[8, \S 1.6]$. Thus

$$
\begin{aligned}
\|\alpha(r)\|_{X_{0}} & =\sum_{x \in \tilde{X}_{0}} Z_{x}\left(\tilde{f}_{\alpha(r)}\right) \\
& =m_{0}+\sum_{x \in X_{0}^{\nu}}\left(Z_{x}\left(\tilde{f}_{\alpha(r)}\right)-\min \left\{Z_{x}\left(\tilde{f}_{\beta}\right) \mid \beta \in H_{1}(\partial M)\right\}\right) \\
& =m_{0}+\sum_{x \in J}\left(Z_{x}\left(\tilde{f}_{\alpha(r)}\right)-\min \left\{Z_{x}\left(\tilde{f}_{\beta}\right) \mid \beta \in H_{1}(\partial M)\right\}\right) \\
& =m_{0}+2 \# J \quad \text { by }(1) \text { above } \\
& =m_{0}+2 \# K,
\end{aligned}
$$

which is what we set out to prove.

\section{Jumps in multiplicities of zeros in the case of $P S L(2, \mathbb{C})$-characters}

For a finitely generated group $\Gamma$ we set $\bar{R}(\Gamma)=\operatorname{Hom}(\Gamma, P S L(2, \mathbb{C}))$. Then $\bar{R}(\Gamma)$ is a complex affine algebraic set (see eg. [5]) and the natural action of $P S L_{2}(\mathbb{C})$ on $\bar{R}(\Gamma)$ has an algebro-geometric quotient $\bar{X}(\Gamma)$. There is a surjective quotient map

$$
\bar{t}: \bar{R}(\Gamma) \longrightarrow \bar{X}(\Gamma)
$$

For each $\gamma \in \Gamma$, the function $\bar{X}(\Gamma) \rightarrow \mathbb{C}$ given by

$$
f_{\gamma}: \bar{X}(\Gamma) \rightarrow \mathbb{C}, \quad \bar{t}(\rho) \mapsto \operatorname{tr}(\rho(\gamma))^{2}-4 .
$$

is regular. By analogy, $\bar{X}(\Gamma)$ is called the set of $P S L_{2}(\mathbb{C})$-characters of $\Gamma$ and $\bar{t}(\bar{\rho})$ will be denoted by $\chi_{\bar{\rho}}$.

A representation $\bar{\rho} \in \bar{R}(\Gamma)$ is called irreducible if it is not conjugate to a representation whose image lies in $\left\{ \pm\left(\begin{array}{cc}a & b \\ 0 & a^{-1}\end{array}\right) \mid a, b \in \mathbb{C}, a \neq 0\right\}$. Otherwise it is called reducible. Two points worth mentioning are (i) the image of an irreducible representation in $\operatorname{PSL}(2, \mathbb{C})$ is either non-abelian or $\mathbb{Z} / 2 \oplus \mathbb{Z} / 2$ and (ii) any two subgroups of $P S L(2, \mathbb{C}$ ) abstractly isomorphic to $\mathbb{Z} / 2 \oplus \mathbb{Z} / 2$ are conjugate.

Let $N \subset P S L(2, \mathbb{C})$ denote the subgroup

$$
\left\{ \pm\left(\begin{array}{cc}
z & 0 \\
0 & z^{-1}
\end{array}\right), \pm\left(\begin{array}{cc}
0 & w \\
-w^{-1} & 0
\end{array}\right) \mid z, w \in \mathbb{C}^{*}\right\}
$$


of $P S L(2, \mathbb{C})$. One of the features which distinguishes the $S L(2, \mathbb{C})$ and $P S L(2, \mathbb{C})$ theories is that when an irreducible $P S L(2, \mathbb{C})$ representation $\rho$ conjugates into $N, P S L(2, \mathbb{C})$ does not act freely on the orbit $\mathcal{O}(\rho)$, but has, rather, $\mathbb{Z} / 2$ isotropy. This fact obliges us to add an extra case to the $P S L(2, \mathbb{C})$ version of Theorem A.

A curve $X_{0} \subset \bar{X}(\Gamma)$ which contains the character of an irreducible representation is called non-trivial. For any such curve there is a unique 4-dimensional subvariety $R_{0} \subset$ $\bar{R}(M)$, invariant under the action of $P S L(2, \mathbb{C})$ which satisfies $t\left(R_{0}\right)=X_{0}[5$, Lemma 4.1].

Theorem 2.1 Fix a slope $r$ on $\partial M$ and consider a non-trivial curve $X_{0} \subset \bar{X}(M)$. Suppose that $x \in X_{0}^{\nu}$ is a point where $\nu(x)=\chi_{\rho}$ for some representation $\rho \in R_{0}$ which is irreducible or has non-abelian image and which satisfyies $\rho(\alpha(r))= \pm I$. Assume $H^{1}(M(r) ; A d \rho)=0$ and $\rho\left(\pi_{1}(\partial M)\right) \neq\{ \pm I\}$.

(1) If $\beta \in \pi_{1}(\partial M)$ and $\rho(\beta) \neq \pm I$, then

$$
Z_{x}\left(\tilde{f}_{\alpha(r)}\right) \geq \begin{cases}Z_{x}\left(\tilde{f}_{\beta}\right)+1 & \text { if } \rho \text { conjugates into } N \\ Z_{x}\left(\tilde{f}_{\beta}\right)+2 & \text { otherwise. }\end{cases}
$$

(2) If $\beta \in \pi_{1}(\partial M)$ and $Z_{x}\left(\tilde{f}_{\alpha(r)}\right)>Z_{x}\left(\tilde{f}_{\beta}\right)$, then $f_{\alpha(r)} \mid X_{0} \neq 0, \rho(\beta) \neq \pm I$, and

$$
Z_{x}\left(\tilde{f}_{\alpha(r)}\right)= \begin{cases}Z_{x}\left(\tilde{f}_{\beta}\right)+1 & \text { if } \rho \text { conjugates into } N \\ Z_{x}\left(\tilde{f}_{\beta}\right)+2 & \text { otherwise }\end{cases}
$$

Proof. Let $R_{0}$ be the unique algebraic component of $\bar{t}^{-1}\left(X_{0}\right) \subset \bar{R}(M)$ for which $\bar{t}\left(R_{0}\right)=$ $X_{0}$ [5, Lemma 4.1]. Then $R_{0}$ has dimension 4 and is invariant under conjugation by $P S L(2, \mathbb{C})$. Arguing as in [6] or $\S 3$ of [2], we can show that the $P S L_{2}(\mathbb{C})$-action on $R_{0}$ admits an analytic 2-disk slice $D$ at $\rho$. When $\rho$ does not conjugate into $N$, the fact that $P S L(2, \mathbb{C})$ acts freely on $\mathcal{O}(\rho)$ implies that $\chi_{\rho}$ has a neighbourhood in $X_{0}$ which is analytically equivalent, by $\bar{t}$, to an open subset of $D$. A proof identical to the one above shows that the theorem holds in this case.

Assume then that $\rho$ conjugates into $N$. In this case our hypotheses imply that $\rho$ is irreducible. Note also that $\rho\left(\pi_{1}(\partial M)\right)$ is diagonalisible and so $Z_{x}\left(\tilde{f}_{\beta}\right)=0$ and $Z_{x}\left(\tilde{f}_{\alpha(r)}\right) \geq$ 1. To see that $Z_{x}\left(\tilde{f}_{\alpha(r)}\right)=1$ when $Z_{x}\left(\tilde{f}_{\alpha(r)}\right)>Z_{x}\left(\tilde{f}_{\beta}\right)$ we first observe that $\chi_{\rho}$ has a neighbourhood in $X_{0}$ which is analytically equivalent to the quotient of $D$ by the action of the $\mathbf{Z} / 2$ isotropy group of $\rho[5, \S 6]$. This action is linear and so $\nu(x)=\chi_{\rho}$ is a smooth point of $X_{0}$ and $T_{x} X_{0}$ may be identified with $H^{1}(M ; A d \circ \rho) /\{ \pm 1\}$. The effect this $2-1$ branching has on the calculation of $Z_{x}\left(\tilde{f}_{\alpha(r)}\right)$ in $\S 1.2 .1$ is to replace the identity $Z_{x}\left(\tilde{f}_{\alpha(r)}\right)=2$ there by $Z_{x}\left(\tilde{f}_{\alpha(r)}\right)=1$ here. This completes the proof.

The following corollary is proven in a manner similar to the derivation of Corollary B from Theorem A. 
Corollary 2.2 Assume the hypotheses of Theorem $C$.

(1) If $\beta \in H_{1}(\partial M) \backslash\{0\}$ and $f_{\beta} \mid X_{0}=0$, then $f_{\alpha(r)} \mid X_{0}=0$.

(2) If $f_{\alpha(r)} \mid X_{0} \neq 0$ and $\beta \in H_{1}(\partial M)$, then

$$
Z_{x}\left(\tilde{f}_{\beta}\right)=\left\{\begin{array}{rr}
Z_{x}\left(\tilde{f}_{\alpha(r)}\right)-2 & \text { if } \beta \in H_{1}(\partial M) \backslash\{n \alpha(r) \mid n \in \mathbb{Z}\} \text { and } \rho \text { does not } \\
& \text { conjugate into } N \\
Z_{x}\left(\tilde{f}_{\alpha(r)}\right)-1 & \text { if } \beta \in H_{1}(\partial M) \backslash\{n \alpha(r) \mid n \in \mathbb{Z}\} \text { and } \rho \text { conjugates } \\
& \text { into } N \\
Z_{x}\left(\tilde{f}_{\alpha(r)}\right) & \text { if } \beta=n \alpha(r) \text { some } n \in \mathbb{Z} \backslash\{0\} \\
\infty & \text { if } \beta=0 .
\end{array}\right.
$$

Culler-Shalen seminorms are defined in the $\operatorname{PSL}(2, \mathbb{C})$ setting $([5])$. We have the following analogue of Theorem C.

Theorem 2.3 Let $M$ be the exterior of a knot in a closed, connected, orientable 3-manifold $W$ for which $\operatorname{Hom}\left(\pi_{1}(W), P S L(2, \mathbb{C})\right)$ contains only diagonalisable representations. Suppose further that there is a non-boundary slope $r$ for which $M(r)$ is a non-Haken small Seifert manifold. Fix a non-trivial curve $X_{0} \subset \bar{X}(M)$ for which $f_{\alpha(r)} \mid X_{0}$ is non-constant. Then

$$
\|\alpha(r)\|_{X_{0}}=m_{0}+A+2 B
$$

where

$$
m_{0}=\sum_{x \in \tilde{X}_{0}} \min \left\{Z_{x}\left(\tilde{f}_{\alpha}\right)\left|\tilde{f}_{\alpha}\right| \tilde{X}_{0} \not \equiv 0\right\},
$$

while $A$, resp. $B$, is the number of irreducible characters $\chi_{\rho} \in X_{0}$ of representations $\rho$ which conjugate, resp. do not conjugate, into $N$ and such that $\rho(\alpha(r))= \pm I$.

\section{Counting the $P S L(2, \mathbb{C})$ characters of small Seifert manifolds}

Throughout this section we shall assume that $W$ is a non-Haken small Seifert manifold whose base orbifold is of the form $S^{2}(p, q, r)$ where $p, q, r \geq 2$ (though see Remark 3.3). It is shown in $[11, \S \mathrm{VI}]$ that $\# H_{1}(W)<\infty$ and that there is a presentation of $\pi_{1}(W)$ of the form

$$
\pi_{1}(W)=<x, y, h \mid h \text { central, } x^{p}=h^{a}, y^{q}=h^{b},(x y)^{r}=h^{c}>
$$


where $\operatorname{gcd}(a, p)=\operatorname{gcd}(b, q)=\operatorname{gcd}(c, r)=1$. It follows that

$$
H_{1}(W) \cong \mathbb{Z} / \operatorname{gcd}(p, q, r) \oplus \mathbb{Z} / f
$$

where $f=\frac{|a q r+b p r+c p q|}{\operatorname{gcd}(p, q, r)}$. Since every reducible character is a character of a diagonal representation, it can be verified that the number of reducible characters in $\bar{X}(W)$ is

$$
\# \bar{X}^{r e d}(W)=\left\lfloor\frac{|a q r+b p r+c p q|}{2}\right\rfloor+ \begin{cases}1 & \text { if } \operatorname{gcd}(p, q, r) \text { is odd } \\ 2 & \text { if } \operatorname{gcd}(p, q, r) \text { is even }\end{cases}
$$

(here $\lfloor\cdot\rfloor: \mathbb{R} \rightarrow \mathbb{Z}$ denotes the "greatest integer less than or equal to" function). This proves part (1) of Proposition D.

Lemma 3.1 Let $\rho \in \bar{R}(W)$.

(1) If $\rho(h)$ is parabolic, then $\chi_{\rho}$ is the character of the trivial representation.

(2) If $\rho(h) \neq \pm I$ and is diagonalisable, then $\rho\left(\pi_{1}(W)\right)$ is diagonalisable.

Proof. If $\rho(h)$ is a parabolic element of $\operatorname{PSL}(2, \mathbb{C})$, then the centrality of $h$ in $\pi_{1}(W)$ can be used to show that each matrix in $\rho\left(\pi_{1}(W)\right)$ is either parabolic or $\pm I$. Thus $\chi_{\rho}$ has the same character as the trivial representation, which proves part (1).

Next suppose that $\rho(h) \neq \pm I$ and is diagonalisable. Then after replacing $\rho$ by a conjugate representation, we may suppose that

$$
\rho(h) \in D=\left\{ \pm\left(\begin{array}{cc}
z & 0 \\
0 & z^{-1}
\end{array}\right) \mid z \in \mathbb{C}^{*}\right\} .
$$

Another application of the centrality of $h$ in $\pi_{1}(W)$ implies that after conjugation either $\rho\left(\pi_{1}(W)\right) \subset D$ or $\rho\left(\pi_{1}(W)\right) \subset N$ and $\rho(h)= \pm\left(\begin{array}{cc}i & 0 \\ 0 & -i\end{array}\right)$. If the second case arises and $\rho(x) \in N \backslash D$, then $\pm I=\rho(x)^{2}$ and so as $\rho\left(x^{p}\right)=\rho\left(h^{a}\right) \in D$, we have $\pm I=\rho(x)^{p}=\rho(h)^{a}$. But then both $a$ and $p$ are even, contrary to our assumptions. Thus $\rho(x) \in D$ and similarly $\rho(y) \in D$. It follows that $\rho\left(\pi_{1}(W)\right) \subset D$ and we are done.

Setting $h=1$ in $\pi_{1}(W)$ induces a surjection of $\pi_{1}(W)$ onto the $(p, q, r)$ triangle group

$$
\Delta(p, q, r)=<x, y \mid x^{p}=1, y^{q}=1,(x y)^{r}=1>.
$$

Note

$$
H_{1}(\Delta(p, q, r)) \cong \mathbb{Z} / \operatorname{gcd}(p, q, r) \oplus \mathbb{Z} / g
$$

where $g=\frac{\operatorname{gcd}(p q, p r, q r)}{\operatorname{gcd}(p, q, r)}$. Then

$$
\# \bar{X}^{r e d}(\Delta(p, q, r))=\left\lfloor\frac{\operatorname{gcd}(p q, p r, q r)}{2}\right\rfloor+ \begin{cases}1 & \text { if } \operatorname{gcd}(p, q, r) \text { is odd } \\ 2 & \text { if } \operatorname{gcd}(p, q, r) \text { is even. }\end{cases}
$$


Since $\bar{X}(\Delta(p, q, r))$ can be identified with $\left\{\chi_{\rho} \in \bar{X}(W) \mid \rho \in \bar{R}(W)\right.$ and $\left.\rho(h)= \pm I\right\}$ while by the lemma above $\left\{\chi_{\rho} \in \bar{X}(W) \mid \rho \in \bar{R}(W)\right.$ and $\left.\rho(h) \neq \pm I\right\} \subset \bar{X}^{\text {red }}(W)$, the set of reducible characters in $\bar{X}(W)$, we have

$$
\begin{aligned}
\# \bar{X}(W) & =\# \bar{X}(\Delta(p, q, r))+\# \bar{X}^{r e d}(W)-\# \bar{X}^{r e d}(\Delta(p, q, r)) . \\
& =\# \bar{X}(\Delta(p, q, r))+\left\lfloor\frac{|a q r+b p r+c p q|}{2}\right\rfloor-\left\lfloor\frac{\operatorname{gcd}(p q, p r, q r)}{2}\right\rfloor .
\end{aligned}
$$

We must therefore compute $\# \bar{X}(\Delta(p, q, r))$.

\section{Proposition 3.2}

(1) The number of reducible characters in $\bar{X}(\Delta(p, q, r))$ is

$$
\left\lfloor\frac{\operatorname{gcd}(p q, p r, q r)}{2}\right\rfloor+ \begin{cases}1 & \text { if } \operatorname{gcd}(p, q, r) \text { is odd } \\ 2 & \text { if } \operatorname{gcd}(p, q, r) \text { is even }\end{cases}
$$

(2) The number of characters in $\bar{X}(\Delta(p, q, r))$ of representations with image a dihedral group of order at least 4 is

$$
\sigma(q, r)\left\lfloor\frac{p}{2}\right\rfloor+\sigma(p, r)\left\lfloor\frac{q}{2}\right\rfloor+\sigma(p, q)\left\lfloor\frac{r}{2}\right\rfloor-2 \sigma(p, q) \sigma(p, r) \sigma(q, r)
$$

where $\sigma(m, n)=1$ if $m, n$ are both even and equals zero otherwise.

(3) The number of characters in $\bar{X}(\Delta(p, q, r))$ is

$$
\left\lfloor\frac{p}{2}\right\rfloor\left\lfloor\frac{q}{2}\right\rfloor\left\lfloor\frac{r}{2}\right\rfloor+\left\lfloor\frac{p-1}{2}\right\rfloor\left\lfloor\frac{q-1}{2}\right\rfloor\left\lfloor\frac{r-1}{2}\right\rfloor+\left\lfloor\frac{\operatorname{gcd}(p, q)}{2}\right\rfloor+\left\lfloor\frac{\operatorname{gcd}(p, r)}{2}\right\rfloor+\left\lfloor\frac{\operatorname{gcd}(q, r)}{2}\right\rfloor+1
$$

Proof. We have already discussed part (1) of the lemma above, so consider part (2). Denote by $D_{n} \subset N \subset P S L(2, \mathbb{C})$ a dihedral group of order $2 n$ and by $C_{n}$ the cyclic, normal subgroup of order $n$ in $D_{n}$. We may assume that $\left(\begin{array}{cc}0 & 1 \\ -1 & 0\end{array}\right) \in D_{n}$. Any representation $\rho \in \bar{R}(\Delta(p, q, r))$ with image $D_{n}$ must send at least two of $x, y, x y$ to $D_{n} \backslash C_{n}$, a set consisting of elements whose square is $\pm I$, and so the corresponding orders amongst $p, q, r$ are even. Thus part (2) of the lemma holds if at least two of $p, q, r$ are odd. On the other hand if, say, $\rho(x), \rho(y) \in D_{n} \backslash C_{n}$, then up to conjugation $\rho(x)=\left(\begin{array}{cc}0 & 1 \\ -1 & 0\end{array}\right)$ and $\rho(x y)$ is a diagonal matrix satisfying $\rho(x y)^{r}= \pm I$. It is easily seen that there are $\left\lfloor\frac{r}{2}\right\rfloor$ characters of such representations onto some $D_{n}$ with $n \geq 2$, and therefore part (2) of the lemma holds if exactly one of $p, q, r$ is odd. To see that it holds when $p, q, r$ are all even, one simply observes that the $\left\lfloor\frac{p}{2}\right\rfloor+\left\lfloor\frac{q}{2}\right\rfloor+\left\lfloor\frac{r}{2}\right\rfloor$ characters of representations of $\Delta(p, q, r)$ onto some $D_{n}$ $(n \geq 2)$ just constructed are distinct except for the three characters of representations with image $D_{2}$. 
Next we prove part (3). Owing to the fact that $\Delta(p, q, r)$ is a quotient of $\mathbb{Z} / p * \mathbb{Z} / q=$ $<x, y \mid x^{p}=1, y^{q}=1>$, we may consider $\bar{X}(\Delta(p, q, r)) \subset \bar{X}(\mathbb{Z} / p * \mathbb{Z} / q)$. In Example 2.1 of [5], it is shown that

$$
\bar{X}(\mathbb{Z} / p * \mathbb{Z} / q)=P \sqcup C
$$

where "ப" denotes disjoint union, $P$ is a finite set of isolated points corresponding to characters of representations which send $x$ or $y$ to $\pm I$, and $C$ is a disjoint union of $\left[\frac{p}{2}\right]\left[\frac{q}{2}\right]$ non-trivial curves. Hence

$$
\# \bar{X}(\Delta(p, q, r))=\#(P \cap \bar{X}(\Delta(p, q, r)))+\#(C \cap \bar{X}(\Delta(p, q, r))) .
$$

A point in $P \cap \bar{X}(\Delta(p, q, r))$ is the character of a representation $\rho \in \bar{R}(\Delta(p, q, r))$ for which $\rho(x)$ or $\rho(y)$ is $\pm I$. For instance if $\rho(x)= \pm I$, then since $\rho(y)^{q}= \pm I$ and $\rho(y)^{r}=\rho(x y)^{r}=$ $\pm I$, we have $\rho(y)^{\operatorname{gcd}(q, r)}= \pm I$. There are $\left\lfloor\frac{\operatorname{gcd}(q, r)}{2}\right\rfloor+1$ such characters and it is quickly verified that

$$
\#(P \cap \bar{X}(\Delta(p, q, r)))=\left\lfloor\frac{\operatorname{gcd}(p, r)}{2}\right\rfloor+\left\lfloor\frac{\operatorname{gcd}(q, r)}{2}\right\rfloor+1
$$

Next we consider $C \cap \bar{X}(\Delta(p, q, r))$. Each component of $C$ is isomorphic to a complex line, parameterised as follows (see [5, Example 2.1]). For each pair $(j, k)$, where $1 \leq j \leq\left[\frac{p}{2}\right]$ and $1 \leq k \leq\left[\frac{q}{2}\right]$, set $\lambda=e^{\pi i j / p}, \mu=e^{\pi i k / q}$, and $\tau=\mu+\mu^{-1}$. There is a curve $C(j, k) \subset \bar{X}(\mathbb{Z} / p *$ $\mathbb{Z} / q$ ) whose points are the characters of the representations $\rho_{z} \in \operatorname{Hom}\left(\mathbb{Z} / p * \mathbb{Z} / q, P S L_{2}(\mathbb{C})\right)$, $z \in \mathbb{C}$, where

$$
\rho_{z}(x)= \pm\left(\begin{array}{cc}
\lambda & 0 \\
0 & \lambda^{-1}
\end{array}\right), \quad \rho_{z}(y)= \pm\left(\begin{array}{cc}
z & 1 \\
z(\tau-z)-1 & \tau-z
\end{array}\right) .
$$

We have $C=\sqcup_{j, k} C(j, k)$. The surjective, regular map

$$
\Psi: \mathbb{C} \longrightarrow C(j, k), \quad z \mapsto \chi_{\rho_{z}}
$$

is an isomorphism unless $j=p / 2$ or $k=q / 2$, in which case it is a $2-1$ map branched over the single point $\chi_{\rho_{\frac{\tau}{2}}}$.

Now $\bar{X}(\Delta(p, q, r)) \cap C=A \sqcup B$ where

$$
A=\left\{\chi_{\rho} \in \bar{X}(\Delta(p, q, r)) \mid \chi_{\rho} \in C \text { and } \rho(x y)= \pm I\right\}
$$

and

$$
B=\left\{\chi_{\rho} \in \bar{X}(\Delta(p, q, r)) \mid \chi_{\rho} \in C \text { and } \rho(x y) \neq \pm I\right\} .
$$

Since a character $\chi_{\rho} \in \bar{X}(\Delta(p, q, r))$ lies in $C$ if and only if $\rho(x) \neq \pm I$ and $\rho(y) \neq \pm I$, we have that $\chi_{\rho} \in A$ if and only if after a conjugation we have

$$
\rho(x)= \pm\left(\begin{array}{cc}
\lambda & 0 \\
0 & \lambda^{-1}
\end{array}\right), \quad \rho(y)= \pm\left(\begin{array}{cc}
\lambda^{-1} & 0 \\
0 & \lambda
\end{array}\right)
$$


for some $\lambda=\exp (i \theta) \neq \pm 1$ such that $\theta \in\left(0, \frac{\pi}{2}\right]$ and $\lambda^{2 p}=\lambda^{2 q}=1$. This is equivalent to requiring $\lambda=\nu^{m}$ where $\nu=\exp \left(\frac{2 \pi i}{\operatorname{gcd}(p, q)}\right)$ and $1 \leq m \leq\left\lfloor\frac{\operatorname{gcd}(p, q)}{2}\right\rfloor$. Thus

$$
\# A=\left\lfloor\frac{\operatorname{gcd}(p, q)}{2}\right\rfloor \text {. }
$$

On the other hand if for some $j, k$ as above and $\rho \in \bar{R}(\Delta(p, q, r))$ we have $\chi_{\rho}=\chi_{\rho_{z}} \in$ $B \cap C(j, k)$, then as $\rho(x y)^{r}= \pm I$, the $(2 r)^{t h}$ power of

$$
\left(\begin{array}{cc}
\lambda & 0 \\
0 & \lambda^{-1}
\end{array}\right)\left(\begin{array}{cc}
z & 1 \\
z(\tau-z)-1 & \tau-z
\end{array}\right)
$$

has trace 2. This implies that

$$
2 i \operatorname{Im}(\lambda) z+\lambda^{-1} \tau=\operatorname{tr}\left(\left(\begin{array}{cc}
\lambda & 0 \\
0 & \lambda^{-1}
\end{array}\right)\left(\begin{array}{cc}
z & 1 \\
z(\tau-z)-1 & \tau-z
\end{array}\right)\right)=2 \cos \left(\frac{2 \pi l}{2 r}\right)
$$

for some $l \in\{1,2, \ldots, r-1\}$. Thus the characters $B \cap C(j, k)$ corresponding to representations of $\Delta(p, q, r)$ are contained amongst $\left\{\chi_{\rho_{z_{1}}}, \chi_{\rho_{z_{2}}}, \ldots, \chi_{\rho_{z_{r-1}}}\right\}$ where

$$
z_{l}=\frac{2 \cos \left(\frac{2 \pi l}{2 r}\right)-\lambda^{-1} \tau}{2 i \operatorname{Im}(\lambda)}
$$

Conversely if $l \in\{1,2, \ldots, r-1\}$, then $\operatorname{tr}\left(\rho_{z_{l}}(x y)\right)= \pm 2 \cos \left(\frac{2 \pi l}{2 r}\right) \neq \pm 2$ so that $\rho_{z_{l}}(x y)^{r}= \pm I$ and therefore $\chi_{\rho_{z_{l}}} \in B \cap C(j, k)$. If $\lambda \neq i$ and $\mu \neq i$ (i.e. $\tau \neq 0$ ), then $\chi_{\rho_{z_{1}}}, \chi_{\rho_{z_{2}}}, \ldots, \chi_{\rho_{z_{r-1}}}$ are mutually distinct, while if either $\lambda=i$ or $\mu=i$, it is easy to check that $z_{r-j}=\tau-z_{j}$. Hence

$$
\#(B \cap C(j, k))= \begin{cases}r-1 & \text { if } 1 \leq j<\frac{p}{2} \text { and } 1 \leq k<\frac{q}{2} \\ \left\lfloor\frac{r}{2}\right\rfloor & \text { if } j=\frac{p}{2} \text { or } k=\frac{q}{2} .\end{cases}
$$

Summing over all the curves $C(j, k)$ shows that

$$
\# B= \begin{cases}\left\lfloor\frac{p}{2}\right\rfloor\left\lfloor\frac{q}{2}\right\rfloor(r-1) & \text { if } p, q \text { odd } \\ \left(\left\lfloor\frac{p}{2}\right\rfloor-1\right)\left\lfloor\frac{q}{2}\right\rfloor(r-1)+\left\lfloor\frac{q}{2}\right\rfloor\left\lfloor\frac{r}{2}\right\rfloor & \text { if } p \text { even, } q \text { odd } \\ \left\lfloor\frac{p}{2}\right\rfloor\left(\left\lfloor\frac{q}{2}\right\rfloor-1\right)(r-1)+\left\lfloor\frac{p}{2}\right\rfloor\left\lfloor\frac{r}{2}\right\rfloor & \text { if } p \text { odd, } q \text { even } \\ \left(\left\lfloor\frac{p}{2}\right\rfloor-1\right)\left(\left\lfloor\frac{q}{2}\right\rfloor-1\right)(r-1)+\left\lfloor\frac{p}{2}\right\rfloor\left\lfloor\frac{q}{2}\right\rfloor\left\lfloor\frac{r}{2}\right\rfloor & \text { if } p, q \text { even. }\end{cases}
$$

Collecting the calculations from above and simplifying yields

$$
\begin{aligned}
\# \bar{X}(\Delta(p, q, r))= & \#(C \cap \bar{X}(\Delta(p, q, r)))+\#(P \cap \bar{X}(\Delta(p, q, r))) \\
= & \# A+\# B+\#(P \cap \bar{X}(\Delta(p, q, r))) \\
= & \left\lfloor\frac{p}{2}\right\rfloor\left\lfloor\frac{q}{2}\right\rfloor\left\lfloor\frac{r}{2}\right\rfloor+\left\lfloor\frac{p-1}{2}\right\rfloor\left\lfloor\frac{q-1}{2}\right\rfloor\left\lfloor\frac{r-1}{2}\right\rfloor+\left\lfloor\frac{\operatorname{gcd}(p, q)}{2}\right\rfloor \\
& +\left\lfloor\frac{\operatorname{gcd}(p, r)}{2}\right\rfloor+\left\lfloor\frac{\operatorname{gcd}(q, r)}{2}\right\rfloor+1,
\end{aligned}
$$

which is what we set out to prove. 


\subsection{Proof of Proposition D}

Part (1) of the proposition has been dealt with above, while part (2) is a consequence of Lemma 3.1 and Proposition 3.2. Finally combining Proposition 3.2 with Identity (2) above yields part (3). This completes the proof of Proposition D.

Remark 3.3 If $W$ is a Haken small Seifert manifold with base orbifold $S^{2}(p, q, r)$ where $p, q, r \geq 2$, then $W$ has first Betti number equal to 1 , and since $\bar{X}\left(H_{1}(W)\right) \subset \bar{X}(W)$, the dimension of $\bar{X}(W)$ is 1 . Nevertheless, Lemma 3.1 remains valid in this context and so $\# \bar{X}^{i r r}(W)=\# \bar{X}^{i r r}(\Delta(p, q, r))$.

\section{References}

[1] L. Ben Abdelghani, 'Espace des représentations du groupe d'un noeud dans un groupe de Lie', Ph. D. thesis, Université de Bourgogne-Dijon, 1998.

[2] S. Boyer, 'On the local structure of $S L(2, \mathbb{C})$-character varieties at reducible characters', preprint, 1999.

[3] S. Boyer, T. Mattman and X. Zhang, 'The fundamental polygons of twisted knots and the $(-2,3,7)$ pretzel knot', Proceedings of Knots '96, World Scientific Publishing Co. (1997), $159-172$.

[4] S. Boyer and X. Zhang, 'Finite Dehn surgery on knots', J. Amer. Math. Soc. 9 (1996), 1005-1050.

[5] — 'Culler-Shalen seminorms and Dehn fillings', Ann. of Math. 148 (1998), 737801.

[6] - 'On simple points of character varieties of 3-manifolds', to appear in the Proceedings of Knots in Hellas 1998.

[7] $\quad$, 'A proof of the finite filling conjecture', preprint, 1999.

[8] M. Culler, C. M. Gordon, J. Luecke and P. Shalen, 'Dehn surgery on knots', Ann. of Math. 125 (1987), 237-300.

[9] M. Culler and P. Shalen, 'Varieties of group representations and splittings of 3-manifolds', Ann. of Math. 117 (1983), 109-146.

[10] W. Goldman, 'The symplectic nature of fundamental groups of surfaces', Adv. in Math. 54 (1984), 200-225.

[11] W. Jaco, Lectures on three-manifolds topology, CBMS Regional Conf. Ser. Math. 43, 1980 . 
[12] T. Mattman, 'The Culler-Shalen seminorms of pretzel knots', Ph. D. thesis, McGill University, 2000.

[13] P. E. Newstead, Introduction to moduli problems and orbit spaces, Tata Institute Lecture Notes, Narosa Publishing House, New Delhi, 1978.

Leila Ben Abdelghani

Faculté des Sciences de Monastir

Université de Monastir

Monastir - 5000

Tunisia

e-mail: leila.benabdelghani@fsm.rnu.tn
Steven Boyer

Département de mathématiques, UQAM

P. O. Box 8888, Station "Centre-Ville"

Montreal H3C 3P8

Canada

e-mail: boyer@math.uqam.ca 